\title{
New outbreaks of polio: WHO issues travel warning
}

\section{Background and epidemiology:} In late May, a child was diagnosed with paralytic poliomyelitis in the Darfur region of Sudan, a country that has not seen the disease in over 3 years. The Global Polio Eradication Initiative warns that the case could be only the beginning of the largest polio epidemic in central Africa in recent years and calls for new efforts to expand vaccine coverage in the ar$\approx$ eas affected. ${ }^{1}$

Polio continues to spread in west and central Africa. So far in 2004, paralytic polio has been diagnosed in 5 times as many children in these regions as in the same period 1 year ago.

$\stackrel{\circ}{\circ}$ Moreover, cases of poliomyelitis have been detected in 10 previously polio-free African countries in the last 12 months. $\mathrm{Al}$ most $90 \%$ of polio cases worldwide now occur in Africa.

Clinical description: Polio, which is caused by an enterovirus with 3 serotypes, is spread mainly by the fecal-oral route, but it may also be transmitted by contaminated milk and other foodstuffs or by airborne droplets. After an incubation period of 7-14 days, during which the virus attaches to specific receptors in the oropharynx, tonsils and intestine, it enters the bloodstream and may ascend nerve fibres, where it destroys motor neurons. About

\section{Vaccination for travellers to polio-endemic regions $^{3,4}$}

\section{Infants and children}

- 4 doses of IPV at 2, 4 and 6-18 months of age and 4-6 years of age

- If accelerated immunization is needed, the interval between doses can be reduced to 4 weeks

- Minimum age for vaccination is 6 weeks

Adults

Adults who have not been vaccinated or whose vaccination status is unknown:

- 3 doses of IPV, the first 2 at an interval of 4-6 weeks and the third 6-12 months later

- If immunization is urgent, then doses can be given at 4-week intervals

- 2 doses will provide some protection, and a single dose is recommended if the above options are not possible. In all cases, the remaining doses should eventually be given if the person remains at risk

Adults who have been vaccinated as children should receive a single booster dose of IPV before departure

Contraindications to IPV include a history of anaphylaxis after previous IPV vaccination or allergies to streptomycin, polymyxin B or neomycin (which may be present in trace amounts in IPV)

IPV = inactivated poliovirus vaccine

$1 \%$ of infections result in paralysis. Aseptic meningitis can also occur. The remaining infections present as a mild nonspecific viral illness. Infected people shed the virus in stools. ${ }^{2}$

Prevention: Oral and inactive (injectable) trivalent vaccines are available. The live oral poliovirus vaccine (OPV) is preferable in areas where fecal-oral transmission is frequent. $\mathrm{OPV}$ is used in current containment initiatives.

Travellers to countries (and adjacent countries) that are experiencing polio outbreaks should be vaccinated before departure with 3 doses of inactivated poliovirus vaccine (IPV). Guidelines for vaccination are shown in the box.

IPV provides intestinal immunity and stimulates the formation of circulating antibodies. Unlike OPV, IPV is not contraindicated in immunodeficient persons, but it may not provide adequate protection.

\section{Aleksandra Mišak CMAJ}

\section{References}

1. World Health Organization. Polio experts warn of largest epidemic in recent years, as polio hits Darfur. Geneva: The Organization. June 2004. Available: http://www.who.int/mediacentre /releases/2004/pr45/en/ (accessed 2004 Jul 5).

2. Krym VF, MacDonald RD. Global efforts to eradicate polio. CMAJ 2004;170(2):189-90.

3. CDC National Center for Infectious Diseases. Poliomyelitis. Traveler's health. Available: www.cdc.gov/travel /diseases/polio.htm (accessed 2004 Jun 5).

4. Health Canada. Poliovirus activity in west Africa; routine immunizations and travel. Available: www.hc-sc.gc.ca /pphb-dgspsp/tmp-pmv/2003/pv-wa_e .html (accessed 2004 Jun 5). 